\title{
Urostomy Perforation
}

National Cancer Institute

\section{Source}

National Cancer Institute. Urostomy Perforation. NCI Thesaurus. Code C78681.

A perforation in the anatomic parts which are used in the construction of a urostomy. 